\title{
Modeling and Experimental Studies of the DIII-D Neutral Beam System
}

\author{
B. Crowley ${ }^{\mathrm{a}}$, J. Rauch ${ }^{\mathrm{a}}$, and J.T. Scoville ${ }^{\mathrm{a}}$ \\ ${ }^{a}$ General Atomics, PO Box 85608, San Diego, California 92186-5608, USA
}

\begin{abstract}
In this paper, we present the results of beam physics experimental and modeling efforts aimed at learning from and building on the experience of the DIII-D off-axis neutral beam upgrade and other neutral beam system upgrades such as those at JET. The modeling effort includes electrostatic accelerator modeling (using a Poisson solver), gas dynamics modeling for the neutralizer and beam transport models for the beamline. Experimentally, spectroscopic and calorimetric techniques are used to evaluate the system performance. We seek to understand and ameliorate problems such as anomalous power deposition, originating from misdirected or excessively divergent beam particles, on a number of beamline components. We qualitatively and quantitatively evaluate possible project risks such as neutralization efficiency deficit and high voltage hold off associated with increasing the beam energy up to $105 \mathrm{keV}$
\end{abstract}

Keywords: Neutral beams, DIII-D, ion source, neutralization

\section{Introduction}

Neutral beam (NB) injection is used on most major magnetically confined fusion devices to provide noninductive heating, current drive and core fueling. Additionally neutral beams are the basis of a number of key plasma diagnostics such as charge exchange recombination spectroscopy (CER), Motional stark effect (MSE) and fast ion D-alpha (FIDA) spectroscopy.

The Neutral Beam system on DIII-D consists of eight ion sources on four beamlines. The basis of the DIII-D NB system is the Common Long Pulse Source (CLPS). The CLPS was originally developed at Berkeley in the 1980's [1-3] and has since been implemented in various configurations on US magnetic fusion devices including TFTR, NSTX and DIII-D. In more recent times a version of the CLPS has been commissioned and operated on the EAST tokamak in Hefei, China [4,5].

Essentially the DIII-D version of CLPS is an $80 \mathrm{kV}$ high perveance, deuterium positive ion based system delivering up to $2.5 \mathrm{MW}$ per source. The ion source is a filament driven magnetic bucket design and the accelerator is a slot and rail tetrode design with vertical focusing achieved through tilted grids.

There are a number of proposals to build on the recent very successful DIII-D off-axis neutral beam (OANB) upgrade that involved tilting a beamline to provide off-axis current drive. One future upgrade proposal includes increasing the injected power and energy by extending the beam pulse length and increasing the beam voltage.

In this paper we report on the progress of beam physics experimental and modeling efforts aimed at learning from and building on the experience of the DIII-D OANB upgrade and other NB system upgrades such as those at JET $[6,7]$.

\section{Modeling and Analysis}

\subsection{Beam upgrade options and constraints}

In considering the most practical choice of upgrade it is assumed that with moderate effort and investment the DIII-D neutral beam power supplies could be capable of operating at $105 \mathrm{kV}, 100$ Amperes.

The present configuration of the neutral beam accelerators is a tetrode design with the same inter-grid spacing on all sources. Originally all the sources operated at beam perveance of $\sim 2.9 \times 10^{-6} \mathrm{~A} / \mathrm{V}^{3 / 2}$. In order to facilitate longer beam shots some sources had their aperture reduced from $12 \times 48 \mathrm{~cm}$ to $10 \times 48 \mathrm{~cm}$ to limit power at the edges of the beam. These reduced aperture sources operate at perveance of $2.5 \times 10^{-6} \mathrm{~A} / \mathrm{V}^{3 / 2}$. Each beam is comprised of 56 slit beamlets. The maximum beam voltage currently used is $83 \mathrm{kV}$.

To minimize power loading on the beamline internal components while simultaneously maximizing power delivered to the tokamak, the beams are operated in the regime where the beamlet divergence is minimized. Minimum beamlet divergence is obtained at the "perveance match" condition.

For slit beams the perveance match condition is approximately [8]

$$
{ }_{b}=\frac{I}{V^{3 / 2}}=0.68 \quad C L
$$

where $\quad C L$ is the Child-Langmuir perveance (CL Perveance).

$$
C L=\frac{4 \quad 0}{9} \sqrt{\frac{2 e}{m}} \frac{a}{d^{2}} \div
$$

where $a$ is half the distance between the rails, $d$ is the effective distance between the grids [9] and the other symbols have their usual meaning. 
In principle, near maximum utilization of enhanced NB power supplies could be achieved giving an optimized perveance beam at $103 \mathrm{kV} / 100$ amperes without changing the accelerator structure. However, since beams are the dominant heating system on DIII-D a key concern is the reliability of the system.

\subsection{Conditioning and reliability}

The propensity for electrical breakdown between the grids of an accelerator can be ameliorated by maintaining well-conditioned grids. With increased beam voltage more conditioning time would be required. While a full discussion of the reasons for electrical breakdown and conditioning strategies is beyond the scope of this paper a general guide for good practice in accelerator design is given by the Kilpatrick limit [10]. The limit refers to electrical breakdown in vacuum (pressures in the range $10^{-3}$ to $10^{-7}$ mbar) between two metal electrodes that have not been especially prepared. It is an empirically derived formula that takes the form:

$$
W E^{2} \exp \frac{K_{1}}{E} \div=K_{2}
$$

where $W$ is the maximum possible ionic energy in $\mathrm{eV}, E$ is the electric field at the cathode (plasma grid) and the experimentally determined constants $K_{1}=1.7 \times 10^{5} \mathrm{Vcm}^{-1}$ and $K_{2}=1.8 \times 10^{14} \mathrm{~V}^{3} \mathrm{~cm}^{-2}$. Equation (3) can be used to set an "upper limit" on the electric field for a given potential; Fig. 1 shows the graphical solution to the Kilpatrick limit equation that divides the parameter space into two regions, the non-sparking region to the left and the sparking region to the right. Illustrated on the figure are the operating points for a selection of real accelerators. The present operating point for the DIII-D beams labelled (DIIID 83) is well to the left of the limit. Increasing the voltage above $95 \mathrm{kV}$ would bring the operating point beyond limit. As is obvious from the figure, it is possible to run an accelerator above the limit. The JET $80 \mathrm{kV}$ tetrode and the JET SHC (super high current) tetrode were both operated above the limit with

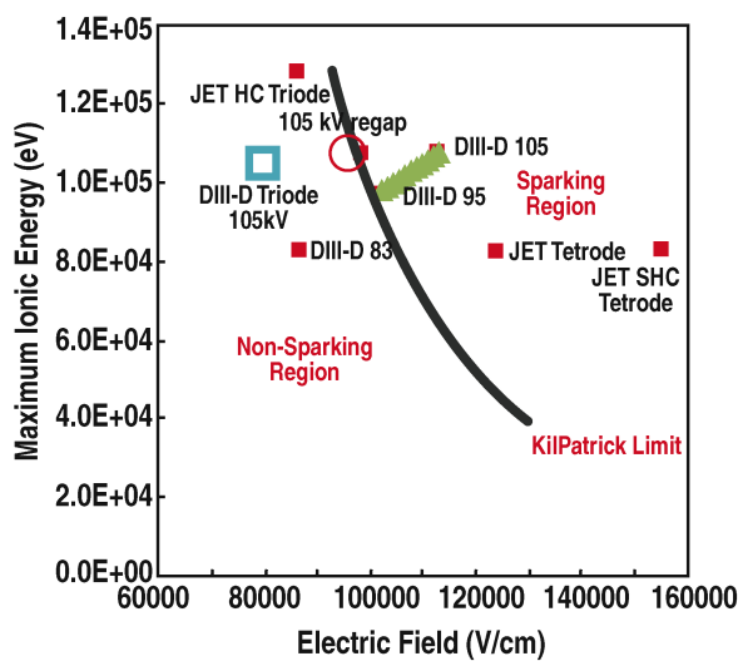

Fig. 1. Kilpatrick limit curve with a number of neutral beam accelerator configurations. the benefit of a dedicated conditioning facility at the JET neutral beam test-bed.

Since a dedicated conditioning facility is not available to DIII-D, the sources and accelerators have to be conditioned in a limited time period before experimental campaigns.

To maintain reliability it is desirable to keep on the low-field side of the Kilpatrick curve. Thus increasing the beam voltage above $95 \mathrm{kV}$ requires that the effective distance between grids in the accelerator be increased. By Eq. (2) if the distance is increased then the $C L$ Perveance and hence the optimal operating current and power is decreased. Therefore re-gapping the accelerator to increase beam voltage above $95 \mathrm{kV}$ requires a careful consideration of the trade-off between energy and power.

\subsection{Accelerator configuration}

One option for increasing both the energy and power is to change the configuration of the accelerator from a tetrode to a triode design. This would be achieved by removing the gradient grid. The triode configuration would offer the advantage of being well on the low field side of the Kilpatrick curve while operating at a voltage of $105 \mathrm{kV}$.

To fully evaluate the impact of removing the gradient grid would require an experimental comparison of the triode and tetrode version of the CLPS.

In preparation for a future experiment the tetrode and triode geometries were numerically simulated using the IGun [11] code. Figure 2 illustrates the accelerator geometry and optimum perveance beam quality. The results indicate that a reasonable quality beam can be extracted from the triode configuration. Beam divergence as a function of perveance (normalized to optimum perveance) is shown in Fig. 3. The simulations show that the perveance curve is steeper in the case of the triode than the tetrode. This would lead to a greater global divergence for the beam due to the fact that not all of the beamlets can be simultaneously optimized in a real source.

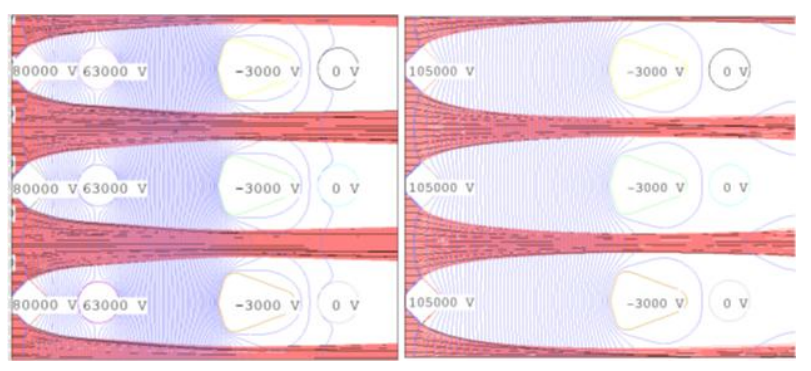

Fig. 2. IGun simulations of tetrode (left) and triode (right) configurations. Blue lines represent equipotential surfaces and red lines show particle trajectories.

The disadvantage of the triode is that the beam optics are poorer with greater beam divergence and greater aberrations in ion trajectories. Mitigation for poorer optics could be achieved by reducing the extraction area 
and/or improving the power handling capabilities of the beamline components.

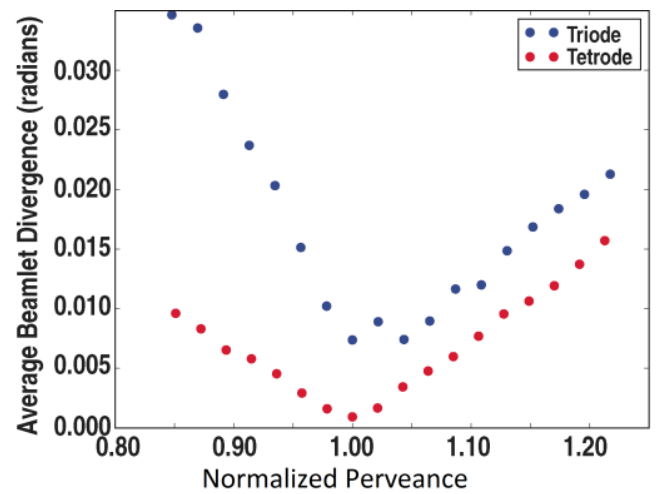

Fig. 3. Perveance curves for tetrode and triode accelerator configurations.

\subsection{Beam power modeling}

A major consideration for any neutral beam upgrade is the power handling capabilities of the existing infrastructure. A significant increase in power or beam on-time would require some components to be changed from inertially-cooled to actively cooled (hypervaptrons or swirl tube). To investigate possible upgrade scenarios, a beam power model has been developed as a tool to determine the power loading on beamline components. The model is similar to the PINI simulator [12] that is used to evaluate ion sources and accelerators at JET. The PINI simulator is the basis of a critical step in the JET QA procedure that ensures that any individual assembled accelerator will produce a beam of sufficient quality that it will transmit through beamline components within allowable limits. Two-dimensional beam profiles at specified distance from the accelerator are generated by summation of 56 individual slit beamlets. Measured accelerator aperture coordinates are used to calculate actual locations (starting points) and orientations (aiming angles) of individual beamlets. The model can be run with measured values to be compared with design values.

Additional steering angles that may be introduced by small offsets in the relative positioning of the grid modules can be incorporated into the model.

Power density distribution of each beamlet is treated separately by assuming: a common shape, common initial waist diameter, variable peak power density and divergence.
An example of the output of the code appears in Fig. 4, showing a 2D beam profile of power density transmitted through and intercepted by the beamline components. The $2 \mathrm{D}$ power density profile can be used as an input to a finite element model to assess the engineering requirements for the beamline.

\subsection{Neutralization efficiency}

A disadvantage of positive ion based neutral beam systems is that the neutralization efficiency decreases as the beam voltage is increased. The theoretical efficiency for a deuterium beam of nominal species composition $85: 10: 5$ at $105 \mathrm{kV}$ is approximately $53 \%$. However, experience from neutral beam upgrades at JET suggest that this theoretical limit is difficult to achieve at higher voltages [13]. Essentially the neutralization efficiency deficit is caused by the heating of the neutralizer gas. The gas is heated due to the interaction of the neutralizer plasma and the neutral gas. The hotter gas leaves the neutralizer and alters the gas density profile along the neutralizer. The altered profile has a lower than optimal line integrated gas density. Amelioration of the phenomenon is not straightforward. The simplest option is to increase the length of the neutralizer, however physical space constraints on the DIII-D beamline rule this out. The option of increasing the gas flow rate has a number of negative consequences that require further modeling and experiments to fully quantify:

1. The gas heating effect is stronger at higher gas densities.

2. Increasing the gas flow at the neutralizer entrance will increase the pressure in the grid region thus making the grids more prone to electrical breakdown.

3. Increasing the overall gas flow into the beamline also increases re-ionization losses further down the beamline.

Figure 5 (a) shows the neutralization efficiency as a function of beam voltage for a number of deuterium beams. Also shown in the figure is the theoretical efficiency for deuterium. The data indicates that up to the present operating limits of $80 \mathrm{kV}$ there is little evidence of neutralization efficiency deficit. However, in figure 5 (b) the same plot is presented for hydrogen and at beam voltages above $75 \mathrm{kV}$ the data indicates the onslaught of the neutralization efficiency deficit similar to JET [13]. Scaling from hydrogen to deuterium suggests that there is a very real possibility of a neutralization efficiency deficit in deuterium at $105 \mathrm{kV}$.
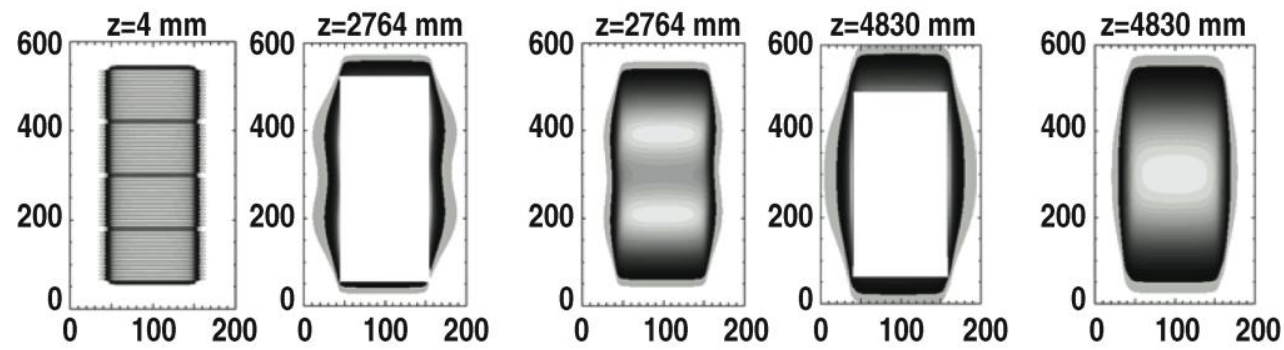
Fig. 4. Simulated transmitted and component intercepted power density profiles at key positions along the beam axis. Accelerator exit, magnet entrance (intercepted and transmitted) and absolute collimator (intercepted and transmitted).
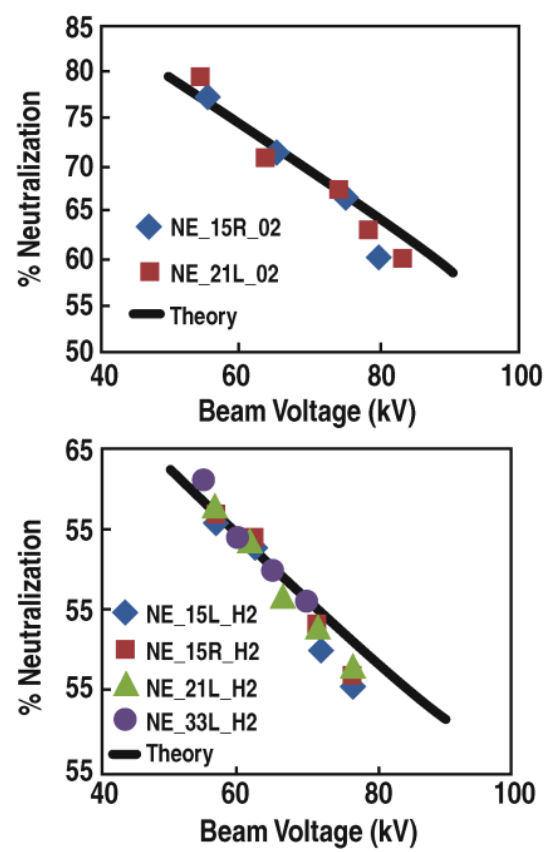

Fig. 5. Measured neutralization efficiency vs. beam voltage for (a) deuterium and (b) hydrogen beams.

A possible partial remedy is to change the neutralizer gas introduction position from the start of the neutralizer to the middle of the neutralizer and increase the gas flow rate. The advantage of this is that the line density profile could be rebalanced so as to maintain the density in the grid region close to its present value while at the same time increasing the flow to the extent where the line density is optimal for neutralization of the beam.

A Monte-Carlo model of the neutralizer and beam line gas dynamics has been used to determine the requirements of a modified gas introduction system. The model, currently restricted to molecular flow, can be used to determine the gas density profile in a rectangular or cylindrical neutralizer geometry. The inputs to the model are gas flow, position of the gas inlet, direction of the initial gas inlet, transparency of the grids, gas baffles, initial gas temperature, final gas temperature and a number of gas heating scenarios. Figure 6 shows the

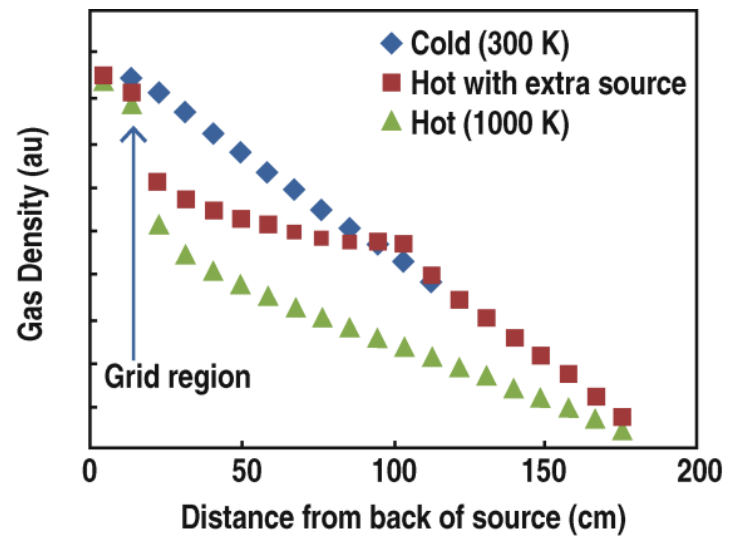

Fig. 6. Neutralizer density profiles. modeled gas density profile for the present configuration for cold gas and hot gas scenarios. Also shown in the figure is a hot gas density profile arising from an additional source of gas positioned at the halfway point of the neutralizer. The results suggest that the line density in the source could be restored to the optimal while simultaneously keeping the gas density in the grid region unchanged.

\section{Conclusion and Future Work}

The presented work provides an insight to some of the issues that will arise from increasing the beam voltage beyond its original design value. Further experimental and modeling work is required to fully assess these issues. Future work proposals on the topic include

1. Experimental measurements of the neutralizer gas temperature.

2. Neutralization efficiency measurements with additional gas source.

3. Testing the CLPS in a triode configuration.

4. Integrating the beamline power model into a quality assurance procedure to enhance the performance and reliability of the NB system.

\section{Acknowledgments}

This work supported in part by the U.S. Department of Energy under DE-FC02-04ER54698.

\section{References}

[1] M.C. Vella, et al., "Prototype testing for the US common long pulse neutral beam source," (LBL-19568) United States.

[2] Wulf B. Kunkel, Rev. Sci. Instrum. 61, 354 (1990).

[3] J.L. Luxon, Nucl. Fusion 42, 614 (2002).

[4] C.D. Hu, NBI Team, "EAST neutral beam injector physical design," Technical Report 09013007, Institute of Plasma Physics, the Chinese Academy of Sciences, Hefei, China (2009).

[5] Chundong $\mathrm{Hu}$, et al., Plasma Sci. Technol. 13, 541 (2011).

[6] D. Ciric, et al., Fusion Eng. Design 86, 509 (2011).

[7] D. Ciric, et al., "Upgrade of the JET neutral beam heating system," Proc. of $19^{\text {th }}$ IEEE/NPSS Symp. on Fusion Engineering, Atlantic, City (2002) p. 140.

[8] T.S. Green, Rep. Prog. Phys. 37, 1257 (194).

[9] A.J.T. Holmes and E. Thompson, Rev. Sci. Instrum. 52, 172 (1981).

[10] W.D. Kilpatrick, Rev. Sci. Instrum. 29, 924 (1957).

[11] R. Becker and W.B. Herrannsfeldt, "IGUN-A Program for the Simulation of Positive Ion Extraction Including Magnetic Fields,” Rev. Sci. Instrum. 63, 2756 (1992). 
[12] D. Ciric, et al., Proc. of $19^{\text {th }}$ IEEE/NPSS Symp. on Fusion Engineering, Atlantic, City (2002) p. 56.

[13] Elizabeth Surrey and Brendan Crowley, Plasma Phys. Control. Fusion 45, 1209 (2003). 